\title{
ORIENTAÇÃO SISTEIMATIZADA DO PACIENTE HIPERTENSO SUBMETIDO A TRATAMENTO AMBULATORIAL
}

\author{
Angela Maria Geraldo Pierin*
}

PIERIN, A.M.G. Orientação sistematizada do paciente hipertenso submetido a tratamento ambulatorial. Rev. Esc. Enf. USP, São Paulo, 20(3):193-205, 1986.

$O$ presente estudo foi realizado em uma unidade ambulatorial que atende exclusivamente pessoas com hipertensão arterial. Teve como objetivos: identificar que orientação os pacientes receberam em relação a medicamentos, dieta, atividades físicas, funo $e$ bebida alcoólica; verificar como se comportam quanto à orientação sistematizada de enfermagem. Os resultados foram distribuidos em freqüências absolutas e relativas e analisados de acordo com os objetivos do estudo.

\section{INTRODUÇÃO}

O controle da pessoa com hipertensão arterial tem sido objeto de estudos, exigindo da equipe de saúde especial atenção e uma programação conjunta, visando a manutenção do hipertenso sob controle.

A hipertensão arterial afeta cerca de $20 \%$ da população adulta, nos paises industrializados (LAURENTI, 1980; LUNA, 1980; SILVA et alii, 1984). Cerca de $90 \%$ dos hipertensos sofrem de hipertensão arterial, essencial, que é uma doença incurável, podendo, entretanto, ser controlada quando submetida a tratamento adequado (CHIAVERINI et alii, 1980).

E comum o hipertenso recusar-se a tomar medicação específica, para o controle da pressão arterial. Paralelamente a isto a hipertensão arterial é uma doença que quando não tratada corretamente pode levar a sérios distürbios cardiacos, cerebrais e da função renal (PODELL, 1976; LAURENTI, 1980).

Em vista destes dados, considera-se de primordial importância manter controlados os niveis tensionais do hipertenso, sendo necessário, para tanto, que o tratamento estabelecido seja seguido satisfatoriamente.

CHIAVERINI et alii (1980) estabeleceu para o tratamento antihipertensivo os seguintes critérios: medidas gerais em que estão incluidos aspectos relacionados ao regime de vida, atividade física e tabagismo; dieta com restrição de sal na alimentação, controle da obesidade e restrição na ingestão de bebida alcoólica, e terapêutica medicamentosa, o principal ponto de apoio para a a redução de pressão arterial.

\footnotetext{
* Enfermeira. Professor Assistente do Departamento de Enfermagem Médico-Cirárgica da Esscola de Enfermagem da USP - disciplina Enfermagem Médico-Cirúrglea.
} 
Para que o tratamento tenha o sucesso desejado a pessoa com hipertensão arterial deverá estar ciente da importância e significado do mesmo, garantindo uma interação efetiva e evitar um possível abandono do tratamento. Assim a educação do hipertenso, em relação à sua saúde, é tida como aspecto relevante.

Pesquisas realizadas comprovaram que a participação da enfermeira junto a programas de orientação de hipertensos contribuiu para o aumento do índice de aderência ao tratamento (WATSON, 1979; ROSENFELD \& SILVERBERG, 1979). Acredita-se que a enfermeira deva atuar junto ao hipertenso no seu papel de educadora, possibilitando um relacionamento de apoio que vise, cada vez mais, a participação ativa do cliente em seu auto cuidado.

Ao considerar a importância do assunto e com a finalidade de levantar dados a respeito, realizou-se o presente estudo com os seguintes objetivos:

1 - identificar quais as orientações recebidas pela pessoa hipertensa em tratamento ambulatorial, quanto a: medicamentos, dieta, atividades físicas, fumo e bebida alcólica;

2 - verificar se a pessoa hipertensa, em tratamento ambulatorial e com orientação sistematizada de enfermagem, atende às orientações recebidas.

\section{MATERIAL E MÉTODO}

O estudo foi realizado em uma unidade ambulatorial de um hospital escola, que atende exclusivamente pacientes com hipertensão arterial.

\section{Poprulação}

Foi constituída por trinta e quatro hipertensos, adultos de ambos os sexos, brasileiros, divididos eqüitativamente em 2 grupos.

Grupo controle: Neste foi observada e anotada quais orientações que o hipertenso recebeu durante a consulta médica em seu primeiro retorno ao serviço. No segundo retorno, após a consulta médica o paciente foi entrevistado, tendo sido verificado se as orientações dadas foram seguidas ou não.

Grupo experimental: Após o primeiro retorno ambulatorial os hipertensos receberam orientações específicas quanto ao tratamento. No segundo retorno procedeu-se de maneira idêntica a adotada com o grupo controle.

\section{Variáveis do estudo}

Variáveis independentes: Consideraram-se idade, sexo, cor, estado civil, escolaridade e ocupação como variáveis que poderiam influenciar a percepção dos clientes quanto à assistência de enfermagem, frente à sua doença, bem como investigar possiveis influências que as mesmas pudessem exercer sobre a população. 
Variáveis dependentes: Neste item foram objeto de estudo as orientações pertinentes a: efeitos e uso dos medicamentos, adequação da dieta, de fumar, ingestão de bebidas alcólicas e atividades físicas.

\section{Coleta de dodos}

Foram utilizados dois formulários (Anexos I e II), sendo o primeiro composto por uma parte inicial de identificação e questões destinadas a averiguar que orientações o cliente recebeu em relação ao tratamento por ocasião do $1^{\circ}$ retorno ambulatorial. No grupo controle os dados foram anotados durante a consulta médica, enquanto que no experimental ao se emitirem as orientações específicas, o preenchimento do instrumento aconteceu concomitantemente às orientações. $O$ segundo formulário (Anexo II) foi formado por questões com o objetivo de se avaliar o cumprimento ou não das orientações recebidas, o que se conseguiu por meio de entrevista realizada com os hipertensos dos 2 grupos, após a consulta médica, no segundo retorno ambulatorial.

A coleta de dados foi efetuada pela autora deste trabalho e por alunas do Curso de Graduação em Enfermagem que faziam parte da equipe de enfermagem responsável pela assistência a hipertensos em tratamento ambulatorial. As alunas de enfermagem foram orientadas e treinadas quanto à metodologia que deveriam utilizar para a obtenção dos dados e quanto ao preenchimento dos formulários, a fim de ser mantida uniformidade na coleta. As entrevistas foram realizadas em local reservado, após a aquiescência do cliente.

\section{Tratamento estatistico}

Os dados obtidos foram expressos em números absolutos e indices percentuais.

\section{RESULTADOS E DISCUSSÃO}

\section{Caracterização da população}

A hipertensão arterial é mais freqüente no adulto de meia idade, embora possa estar presente em qualquer faixa etária (SMITH, 1977; PAGE, 1983; HABERMANN, 1983). Os resultados deste estudo revelaram que a faixa etária predominante foi a de 40 a 60 anos $(70,6 \%)$, o que coincide com dados da literatura.

O hipertenso, que apresenta sintomatologia decorrente da hipertensão arterial, poderá ter a sua produção como trabalhador afetada ou ser afastado temporária ou definitivamente do trabalho, mesmo em idade não compativel com aposentadoria.

A hipertensão arterial incide mais em mulheres, apesar de existir evidência de que, na população em geral, ela seja igualmente distribuída para ambos os sexos (NUSSENZVEIG, 1980). No presente estudo encontrou-se uma predominância do sexo feminino $(67,6 \%)$. 
No que se refere à raça, vários autores apontaram maior prevalência da hipertensão arterial na raça negra (Organização Mundial de Saúde, 1978; CARVALHO, 1982; RIBEIRO et alii, 1982). Os resultados encontrados demonstraram que a maior parte dos hipertensos que compôs a amostra era de cor branca $(41,2 \%)$, sendo os de cor preta $(35,3 \%)$ a parda $(20(6 \%)$ também significativos, o que pode ser explicado pela possível influência do fator genético na incidência da hipertensão arterial.

Quanto ao estado civil verificou-se que a maioria $(70,6 \%)$ era casada, o que condiz com a faixa etária predominante; vindo a seguir os viúvos $(14,7 \%)$, desquitados $(11,8 \%)$ e solteiros $(2,9 \%)$.

Em relação a escolaridade e hipertensão arterial, estudos citados por CURRY \& JENKINS (1979) indicaram que há uma tendência para menor prevalència da doença com o aumento do nível de escolaridade. $\mathrm{Na}$ população estudada evidenciou-se um grande número de pacientes com baixa escolaridade, analfabetos $(23,8 \%)$, instrução primária $(64,7 \%)$ e secunciária $(11,8 \%)$. Esta variável merece atenção especial, pois poderá influenciar no grau de compreensão das orientações emitidas aos hiper'tensos.

Ao se correlacionar hipertensão arterial e ocupação, estudos revelaram maior prevalência em pessoas com atividades ocupacionais não especializadas (CURRY \& JENKINS, 1977; CARVALHO, 1982). Os dados encontrados indicaram que a maioria $(67,6 \%)$ exercia atividade manual não especializada, como "prendas domésticas" e empregada doméstica; $(29,5 \%)$ dedicavam-se a ocupação manual especializada, como pintor, pedreiro, motorista, e os demais, $(2,9 \%)$ a atividades não manuais como por exemplo escriturário, vendedor, dentre outros.

\section{Orientaçöes quanto a mes medicamentos anti hipertensivos}

A hipertensão arterial, pode ser controlada na maioria dos pacientes, pois, atualmente dispõe-se de grande número de medicamentos com as mais variadas ações farmacodinâmicas anti hipertensivas. Um fator primcrdial a ser considerado, também, é o grau de hipertensão do paciente. Vários autores são unânimes ao afirmarem que a terapêutica medicamentosa é o principal ponto de apoio no controle da pressão arterial (BREST, 1981; CHIAVERINI et alii, 1980; SILVA et alii, 1984).

SHOOF (1980) citou algumas perguntas feitas por hipertensos:

- "Por quanto tempo devo tomar os remédios?"

- "O que posso fazer para controlar a pressão?"

- "O que fazer para lembrar de tomar os remédios?"

Com base nestas indagações acredita-se que orientação, quanto à terapêutica medicamentosa instituída, poderá auxiliar o hipertenso a controlar as cifras tensionais elevadas. Os dados deste estudo evidenciaram que todos os pacientes receberam pelo menos uma orientação neste aspecto, seja pelo médico, seja pela enfermeira. 
$\mathrm{Na}$ presente investigação as orientações relativas aos remédios concentraram-se 3 tópicos: dose, horário e efeito esperado. A dose do medicamento a ser tomado foi esclarecido a todos os pacientes dos 2 grupos em estudo. Quanto ao horário, todos aqueles pertencentes ao grupo experimental e $40,0 \%$ do grupo controle receberam esclarecimentos nesse aspecto, o mesmo não ocorrendo no que diz respeito ao efeito dos medicamentos, onde somente parte dos pacientes do grupo experimental $(27,2 \%)$ foi orientada. necessário, para a redução da pressão arterial, que os medicamentos sejam tomados na dose certa e no horário recomendado. Para que isto aconteça satisfatoriamente, deve haver adaptação entre o esquema terapêutico e os hábitos de vida do hipertenso. A enfermeira deverá lançar mão de recursos que poderão auxiliar, tais como: correlacionar a ingestão dos medicamentos com atividades rotineiras, como por exemplo o horário das refeições, o deitar e o levantar-se, o ato de escovar os dentes, dentre outros; orientar para manter os remédios em local visivel ou separar em doses que poderão ser levadas para o local de trabalho. Enfim todos os esforços deverão ser dirigidos para que se consiga interação efetiva do hipertenso com o seu tratamento. Ao se orientar sobre os efeitos da terapêutica anti hipertensiva devem-se abordar principalmente os aspectos benéficos do tratamento, esclarecendo que caso ocorra qualquer sintomatologia desagradável, o cliente não deve deixar de tomar os remédios e sim procurar o médico; a abordagem de sintomas indesejáveis numa fase inicial poderia dissuadir o hipertenso do tratamento.

PODELL (1974) esclareceu que “... o comportamento saúde individual pode ser influenciado pela percepção de barreiras e obstáculos associados ao plano de tratamento proposto. Essas barreiras podem ser internas como as defesas psicológicas ou externas como o custo do trata mento". A maioria dos pacientes $(68,7 \%$ do grupo experimental e $53,3 \%$ do grupo controle) referiu não ter condições financeiras para a compra dos medicamentos, fator este que poderia influenciar abandono do tratamento.

BULLENN (1980) estabeleceu pontos a serem seguidos na orientação sobre a terapêutica medicamentosa anti hipertensiva, salientando que 0 conhecimento do nome dos remédios é um fator a ser considerado. Observou-se que a maioria dos pacientes, tanto do grupo controle como do experimental $(60,0 \%$ e $75,0 \%$ respectivamente), sabia referir o nome dos remédios que estavam tomando. A não referência do nome dos medicamentos seria decorrente da baixa escolaridade predominante na população em estudo.

O estabelecimento de horário para a ingestão dos medicamentos pode ser um ponto favorável para a obtenção da adesão do hipertenso ao tratamento. Nesta investigação verificou-se a ocorrência deste fato em $73,3 \%$ dos hipertensos do grupo controle, enquanto que no experimental, onde a orientação foi dada pela enfermeira, este índice elevou-se para $87,5 \%$. Aqueles do grupo experimental, que não tinham horário determinado para tomar seus remédios, dedicavam-se a atividade ocupacional 
extra domiciliar, fato que estaria ligado à inadequação entre horário e ingestão dos medicamentos. Apenas um hipertenso do grupo controle tinha atividade acupacional fora de sua moradia.

Os dados deste estudo evidenciaram que nos dois grupos houve pacientes que deixaram de tomar seus medicamentos $(43,8 \%$ no experimental e $53,3 \%$ no controle); ao se identificarem os motivos observou-se uma diversidade de razões, sendo as mais citadas: esquecimento, acabou o remédio e não tinha dinheiro para a compra de outro, sentiu alterações desagradáveis e deixou de tomar o remédio para fazer exames.

PODELL (1976) enfatizou a importância da obtenção de dados tais como efeitos colaterais do medicamento, recusa e temores quanto ao tratamento, que necessitam ser considerados pelo orientador para que ocorı'a melhor aceitação do tratamento instituído. Ao se identificarem possíveis alterações decorrentes da terapêutica medicamentosa, neste estudo, verificou-se que estas foram menos freqüentes $(40,4 \%)$ no grupo onde houve orientação sistematizada feita pela enfermeira. As alteraçōes mais citadas foram: aumento da freqüencia urinária, anorexia, sedação, cansaço, tontura, dentre outras. A enfermeira, ao orientar o hipertenso sobre os medicamentos, deverá ter em mente que cada pessoa reage de modo específico em face da terapêutica anti hipertensiva, sendo oportuno realçar o objetivo principal do tratamento, que é a redução dos níveis tensionais. Muitos hipertensos, quando não esclarecidos, abandonam o tratamento ao primeiro problema; as orientações quanto à fase inicial de adaptação da droga ao organismo é ponto relevante para uma ação satisfatória.

\section{Orientações quanto à dieta}

No tratamento da hipertensão arterial, a dieta é um elemento de grande valia, principalmente no que se refere à quantidade de sal e teor calórico. O ideal seria restringir o sal e a ingestão de alimentos ricos em hidrato de carbono e lipidio (CHIAVERINI et alii, 1980; GAMARSKI, 1981; SILVA et alii, 1984). A maioria da população estudada teve uma dieta especial, como prescrição médica, seja hipossódica $(41,2 \%)$, hipocalórica $(17,6 \%)$ ou assódica $(3,0 \%)$. Observou-se também que, tanto no grupo controle, como no experimental $(70,6 \%$ e $88,2 \%$ respectivamente), os hipertensos receberam orientaçōes especificas quanto à dieta, tanto da enfermeira como do médico.

Ao dar as orientações pertinentes à dieta, procurou-se centralizá-las na diminuição do sal, tanto no preparo como na ingestão de alimentos ricos neste ingrediente. Os hipertensos do grupo experimental foram instruidos pela enfermeira a diminuir o sal no preparo dos alimentos; a evitar a sua adição durante as refeições, o que pode ser conseguido com a não permanência do saleiro na mesa; a não ingerir alimentos ricos em sal como peixes, carnes salgadas, defumados, queijo e outros laticínios com sal, condimentos não naturais, e a restringir alimentos hipercalóricos como massas, doces, batatas e gorduras, visto que a obesidade agrava a condição de hipertensão arterial. Paralelamente, os hiper- 
tensos receberam esclarecimentos quanto às possiveis maneira de realçar o sabor da dieta, usando temperos naturais, como salsa, cebola, orégano, manjericão, limão, dentre outros; foj-lhes ensinado que, ao preparar as refeições, deveriam utilizar sal em apenas um tipo de alimento, visto que, ao saboreá-los em conjunto, aquele sem sal passaria desapercebido.

ROGUS (1981) estabeleceu pontos que devem ser seguidos para se conseguir a aderência do hipertenso ao tratamento dietético, enfatisando o papel da enfermeira como educadora; para tanto ela deverá levantar os conhecimentos que o hipertenso tem a respeito do assunto, identificar as suas condiçōes para a compra e preparo dos alimentos, verificar crenças de saúde, com o objetivo de obter ampla participação do hipertenso em seu tratamento. No presente estudo procurou-se identificar, junto ao paciente, qual a composição de sua dieta rotineira, antes de se dizer-lhe o que ele deveria ou não restringir na alimentação.

$\mathrm{Na}$ segunda fase do estudo, onde se verificou o cumprimento ou não das orientações dadas anteriormente, observou-se que a maioria da população $(82,3 \%)$ referiu ter restringido ou retirado totalmente o sal da alimentação.

Aqueles que não alteraram a quantidade de sal na dieta talvez não estivessem motivados o suficiente para seguir as orientações, ou estas não foram explícitas o necessário para compreensão satisfatória, ou ainda, poderiam ter sido em número excessivo para um só momento.

Ressalta-se que a população estudada foi orientada apenas uma vez em seu primeiro retorno ao ambulatório, seja aleatoriamente pelo médico, no grupo controle, ou sistematicamente pela enfermeira, no grupo experimental. Ao se orientarem os hipertensos do grupo experimental, procurou-se dar os esclarecimentos de forma clara, objetiva e parceladamente sobre terapêutica medicamentosa, dieta, atividade física, hábito de fumar e ingestão de bebidas alcoólicas.

SECHEHAYE (1982) afirmou: “... de tudo que se diz a um paciente, só um quarto é ouvido e só um décimo é compreendido". Acredita-se, portanto, que uma orientação correta e individualizada tranquiliza o paciente e o motiva para o auto cuidado.

\section{Orientação quanto ao hábito de fumar}

A hipertensão arterial e o hábito de fumar também se correlacionam, visto que o fumo apresenta relações estreitas com a aterosclerose, que é um elemento agravante da hipertensão arterial (CHIAVERINI et alii, 1980; CASTELLI, 1982; SILVA et alii, 1984).

Neste estudo verificou-se que a maioria da população não tinha o hábito de fumar (76,5\% no grupo controle e $64,7 \%$ no experimental). Após orientação dos fumantes do grupo experimental, constatou-se que apenas um não alterou tal hábito, sendo que os demais abandonaram o fumo ou reduziram o número de cigarros fumados por dia. Já no grupo controle não se observaram modificações relacionadas ao hábito de fumar. Ao 
mesmo tempo convém lembrar que a retirada total do fumo, no hipertenso, poderia causar-lhe certa ansiedade, que indiretamente também contribuiria para a elevação dos níveis tensionais.

\section{Orientações quanto wo hábito de ingerir bebida alcoólica}

No que diz respeito à ingestão de bebidas alcoólicas CHIAVERINI et alii (1980) destacaram que em estudo realizados não se encontrou relação significativa entre o uso de bebidas alcoólicas em doses moderadas e a hipertensão arterial. Apenas um hipertenso do grupo controle citou ingerir bebida alcoólica regularmente, não recebeu orientações quanto a este fato e não apresentou mudança em tal hábito.

\section{Orientação quanto às atividades físicas}

O hipertenso em grau leve ou moderado não necessita restringir as suas atividades físicas, a não ser em caso de eventuais complicações. A atividade física exercida de forma sistematizada poderia até trazer-lhe benefícios, sendo que o repouso regular também influenciaria satisfatoriamente sua saúde (CHIAVERINI et alli, 1980). Neste estudo limitou-se a instruir os hipertensos quanto a atividade física em excesso, pois a recomendação de uma atividade programada implicaria em controle médico específico. Todos os que foram orientados pela enfermeira referiram ter restringido exercícios físicos como trabalhos domésticos, subir escadas, carregar peso, algumas atividades esportivas e terem feito repouso pelo menos uma vez ao dia.

A hipertensão arterial pode ser controlada quando o tratamento proposto for seguido. O hipertenso, principalmente o assintomático, se recusa muitas vezes a aceitar o tratamento, principalmente quando surgem efeitos colaterais indesejáveis com a introdução do esquema terapêutico medicamentoso.

As crenças de saúde também podem influenciar na adesão ou não do hipertenso ao tratamento. JOHNSON (1979) citou estudos onde foi verificado que $98 \%$ dos pacientes acreditavam que a sua pressão arterial estava elevada somente na vigência de sintomas como cefaléia e mal estar, $72 \%$ relataram que quando estavam calmos a pressão arterial diminuia, $77 \%$ aceitavam que deveriam se controlar e $39,5 \%$ sabiam referir quando havia elevação das cifras tensionais.

Acredita-se que a orientação do hipertenso acerca de tratamento, doença, complicações decorrentes dos níveis pressóricos elevados seria uma forma de mantê-lo aderente ao tratamento, sendo a enfermeira a profissional em condições de desenvolver satisfatoriamente esta atividade. ROSENFELD \& SILVERBERG (1979) realizaram estudos evidenciando que, com a introdução de enfermeiras na equipe de atendimento a hipertensos, o controle dos mesmos elevou-se de $39,2 \%$ para $72,8 \%$. WEBB (1980) também salientou que a atuação da enfermeira junto a estas pessoas, só poderia beneficiá-las. GIORGI et alii (1984) apontaram que com 
a atuação da enfermeira, em uma unidade ambulatorial de diagnóstico e tratamento de hipertensão arterial, os índices de persistência ao tratamento se elevaram.

Em face destes fatos considera-se a enfermeira como elemento catalizador dentro da equipe de saúde, que dedica todos os esforços à orientação efetiva do hipertenso, tendo como ponto principal o direcionamento para o auto cuidado pelo cliente. Outro ponto relevante a ser destacado é que antes que se estabeleçam programas específicos de orientação, conheçam-se as reais necessidades da população, para que se possa implementar e adequar atividades que vão de encontro às expectativas das pessoas. Desta forma, a interação estabelecida terá uma base sólida, conseguindo-se atingir os objetivos propostos.

\section{CONCLUSOES}

O presente estudo conduziu às seguintes conclusões:

- as orientações quanto à terapêutica medicamentosa foram concentradas em maior número na dose, seguidas de horário e efeito dos medicamentos;

- quanto à dieta as orientações recaíram em diminuir o sal de alimentação e restringir a ingestão de massas e gorduras;

- as orientações referentes às atividades físicas se concentram em diminuir exercícios físicos como trabalhos domésticos, subir escadas, carregar peso e atividades esportivas;

- os pacientes fumantes foram orientados diminuir o número de cigarros mumados por dia ou abandonar tal hábito;

- no grupo experimental, onde houve orientação sistematizada feita pela enfermeira, foi maior o número de hipertensos que sabiam citar o nome de seus remédios, tinham horário determinado para tomar seus medicamentos e referiram menor número de alterações quanto à terapêutica medicamentosa;

- os dois grupos em estudo se comportaram de maneira semelhante em relação ao cumprimento das orientações dietéticas;

- no tocante ao hábito de fumar, somente no grupo experimental houve hipertensos que apontaram terem alterado este hábito;

- no grupo experimental houve maior número de pessoas que relataram terem diminuido suas atividades físicas.

PIERIN, A.M.G. Systematic orientation of outpatients with arterial hypertension.

Rev. Esc. Enf. USP, São Paulo, 20(3):193-205, 1986.

This study was made in an outpatient treatment unit, with two groups of the patients with arterial hypertension and the objective was to identify the orientations about medications, diet, physical activity, smoke and drink alcoholic and to verify the reaction of the clients to systematic orientation by nurses. The results abtained had been presented in the tables distributed in the absolute and relative frequencies and analysed forward to the objectives of the study. 


\section{REFERENCIAS BIBLIOGRAFICAS}

BREST, N.A. Conduta atual no tratamento clínico da hipertensăo arterial. Temas mod., såo Paulo, 5(6):139-43, jun. 1981.

BULLEN, M.V. What patients with hypertension should known about theirs medications. Drugs, Victoria, 19:373-79, 1980.

CARVALHO, J, Hipertensåo arterial. In: 1982. cap. 1 , p. $3-4$; cap. 7 , p. $108-96$.

Doenca hipertensiva. Săo Paulo, Atheneu,

CASTELLI, W.D. Hypertension: a perspective from the Framinghan experience. In: SLEIGHT, P. \& FREIER, E. Hypertension: cardiology. London, Butterworths, Scientific, 1982, p. 1-19.

CHIAVeriNI, R. et alii . Hipertensão arterial. In: - Doença hipertensiva. Săo Paulo, Atheneu, 1980 . cap. 1 , p. 3-4; cap. 7 , p. 108-96.

CURRY, C.L. \& JENKINS, R.R. Hipertensão nos negros, tratamento multifatorial. Hipertensão pesquisa. São Paulo, 3:2-15, jun. 1979.

GamarsKI, J. Doença hipertensiva. Temas med. São Paulo, 5(6):140-2, jun. 1981.

GIORGI, D.M. et alil. Aderência ao tratamento em hipertensão arterial: intluéncia de variáveis estruturais e de estratégias que visam sua melhoria. In: CONGRESSO BRASILEIRO DE NEFROLOGIA, 120, Salvador, 21-25, out. 1984.

HABERMANN, F. et alii. Níveis pressóricos e prevalência de hipertensão arterial em trabalhadores rurais. In: CONGRESSO PAULISTA DE SAODE PUBLICA, 20, CONGRESSO NACIONAL DA ABRASCO, 19, Săo Paulo, 17-21 abril, 1983. (mimeografado).

JOHNSON, S.S. Health believes of hypertensive patients in a family medicine residency. J. fam. pract., New York, 9(5):877-83, 1979.

LAURENTI, R. Epidemiologia da hipertensão arterial. In: Chiaverini, R. et alii. Doença hipertensiva. São Paulo, Atheneu, 1980. cap. 3, p. 66-87.

LUNA, R.L. Epidemiologia da hipertenaão arterial. In: SIMPOSIO INTERNACIONAL SOBRE HIPERTENSAO ARTERIAL E SUAS COMPLICACóSS. São Paulo, ago. 1980. p. 21-33.

NUSSENZVEIG, I. Hipertensão essencial - manifestações clínicas, evoluçăo e prognóstico. In: Chiaverini, R. et alii. Doenca hipertensiva. Săo Paulo, Atheneu, 1980. cap. 5, p. 117-47.

PAGE, B.L. Epidemiologic evidence on the etiology of human hypertension and its possible prevention. Amer. Hearth. J., Saint Louis, 91(4):527-34. Apr. 1976.

PODELL, R.N. Manual do mécuico sobre controle do paciente na hipertenaão arterial. Såo Paulo, Merck Sharp Dohme do Brasil, 1976. $35 \mathrm{p}$.

RIBEIRO, M.D.R. et alii. Prevalência da hipertensão arterial na força de trabalho da Grande São Paulo: influência da idade, sexo e grupo étnico. Rov. Ass. med. bras. São Paulo, 28(9/10):209-11, set./out. 1982.

ROGUS, S.D. High blood pressure treatment: medication, diet and the rols of the occupational health nurse. Occup. health nurs., Thorofore, 29(11):16-21, Nov. 1981.

ROSENFELD, J.B. \& SILVERBERG, D.S. Status of hipertension treatment in Israel. Israal J. Med. Sci., Tel Aviv, 15(12):1014-16, Dec. 1979.

SHOOF, C.S. Hypertension: common questions patients ask. Amer. J. Nurs., New York, $80(1): 926-7$, May 1980.

SILVA, H.B. et alii. Hipertensăo arterial. In: Marcondes, M. et alii. Clínica médica propedêutica e fisiologia. Rio de Janeiro, Guanabara Koogan, 1984. cap. 21, p. 838-62.

SMITH, W. McF. Epidemiology of hypertension. Med. clin. North Amer., Philadelphia, 61:467-36, 1977 .

WATSON, D.S. Health education for hypertensive patients. Aust. fam. physician, Sidney, 8:315-20, Mar. 1979.

WEBB, P.A. Effectiveness of patient education and psychosocial counseling in promotion compliance and control among hypertension patients, J. fam. pract., New York, 10:(6):104755, 1980.

WORLD HEALTH ORGANIZATION. Arterial hypertension. Geneva, 1978. p. 47-9. (Technical Report Series). 


\section{ANEXO I}

FORMULARIO

No

DATA

\section{I - IDENTIFICAGAO}

Nome:

Idade: ..........; Sexo: $\mathbf{M}() ; \mathbf{F}() ; \operatorname{Cor} \mathbf{B}() ;() ; \mathbf{d}()$

Estado Civil: solteiro ( ) casado ( ) viuvo ( )

$$
\text { desquitado ( ) divorciado }(,) \text { amasiado ( ) }
$$

Escolaridade: analfabeto ( ) primária C ( ) ginasial C ( ) colegial O ( ) universitário $\left.\mathbf{~} \mathbf{I}_{(}\right)$

Ocupaçăo:

Procedencia:

\section{II - 1* FASE}

\section{PARTE A}

1 - Medicamentos prescritas no Serviço Ambulatorial (cópia do prontuárto)

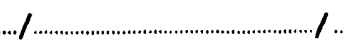
1

2 - Foi orientado sobre as efeitos e maneira de usar os medicamentos que irá tomar? ( ) Sim ( ) Năo

Quem orientou: ( ) médico ( ) enfermeira ( ) outros:

3 - Quais as orientaços recebidas quanto aos medicamentos:

( ) dase díria a ser tomada

( ) horário para tomar cada medicamento

( ) efeito desejado de cada medicamento

( ) outros quais:

4 - Tem poder aquisitivo próprio para comprar as medicaços, se não forem dadas pelo Serviço Ambulatorial?
( ) Sim
( ) Nåo
Como adquire as medicações?

\section{PARTE B}

5 - Dieta prescrita pelo médico:

6 - Foi orientado sobre a dieta que deverá seguir? ( ) Sim ( ) Não Quem orientou: ( ) médico ( ) enfermeira ( ) outros:

7 - Quais as orientaçoes dadas a respeito da dieta?

( ) retirar totalmente o sal no preparo da dieta

( ) diminuir o seal no preparo da dieta

( ) nåo comer alimentos ricos em sal (carnes, peixes, aves e derivados salgados e/ou enlatados, laticínios com sal, frios, moluscos, etc.)

( ) manter dieta normal

( ) outros quais: 


\section{PARTE O}

8 - Tem hábito de fumar ( ) Sim ( ) Nåo

Quantas cigarros por dia?

9 - Foi orientado para diminuir o número de cigarros por dia?

( ) Sim ( ) Não

10 - Tem o hábito de ingerir bebidas alcoslicas diariamente?

( ) Sim ( ) Nâo Quanto? ............................ dia

11 - Foi orientado para diminuir a ingestão de bebidas alcoblicas?

( ) $\operatorname{Sim}($ ) Năo Quem orientou: ( ) médico ( ) enfermeira

( ) outros quem?

\section{PARTE D}

12 - Fol orientado quanto às atividades físicas em excessa?

( ) $\operatorname{Sim}$ ( ) Não Quem orientou: ( 1) médico ( ) enfermeira

$$
\text { () outros quem? }
$$

13 - Quais as orientaçóes recebidas quanto às atividades físicas?

( ) diminuir exercícios físicos como trabalhas domésticos, subir escadas ou ladeiras, carregar peso.

( ) diminuir as atividades desportivas como futebol, natação, ginástica.

( i) manter repouso pelo menos em um período durante o dia.

( ) outros quais: 


\section{FASE}

\section{PARTE A}

1 - Que medicamentos toma para tratamento da Hipertensão Arterial?

1 2 3 4

2 - Tem horário e doses estabelecidas para tomar os medicamentoss?

( ) Sim - Quais? 1 ........ ( ) manhã (6-12h) 2 ........ ( ) manhã (6-12h)

( ) Não ( ) noite $(18-6 \mathrm{~h}) \quad$ ( ) noite (18-6h)

3 - Desde o inicio do tratamento, houve algum periodo em que delxou de tomar os medicamentos?

( ) Sim ( ) Não Por quêe?

4 - Sentiu alguma alteração quando começou a tomar os medicamentos? ( ) Sim ( ) Não Quais?

\section{PARTE B}

5 - Quanto aos cuidados com a dieta:

( ) manteve sal em todos os alimentos

( ) manteve sal em apenas alguns alimentos

( ) manteve sal em apenas um tipo de alimento

( ) outros

6 - Retirou da dieta alimentos ricos em sal?

( ) carnes, aves, peixes e derivados, salgados e/ou defumados

( ) queijos e outros laticínias com sal

( ) alguns legumes como beterraba, vagem, cenoura, aipo, mostarda, espinafre, chucrute

( ) condimentos com sal

( ) outros

\section{PARTE C}

7 - Quanto ao hábito de fumar:

( ) abandonou totalmente

( ) diminuiu o número de cigarros fumados por día

( ) não alterou o hábito

8 - Quanto à ingestão de bebidas alcoolicas:

() abandonou totalmente o hábito

( ) diminuiu a ingestão por dia

( ) não alterou o hábito

\section{PARTE D}

9 - Quanto às atividades físicas:

( ) restringiu exercícios físicos como trabalhos domésticos, subir escadas e ladeiras, carregar peso.

( ) restringiu atividades desportivas como futebol, natação, ginástica.

( ) repousar pelo menos em um periodo do dia

( ) não alterou seus hábitos.

( ) outros 